\title{
LA SATISFACTION DU CONSOMMATEUR SOCIALEMENT RESPONSABLE : UNE ÉTUDE EMPIRIQUE AU MAROC
}

\section{SOCIALLY RESPONSIBLE CONSUMER SATISFACTION: AN EMPIRICAL STUDY IN MOROCCO}

\author{
Carlos Filipe Inglês da SILVA ${ }^{1}$ et Ben Bachir SÂAD ${ }^{1}$ \\ ${ }^{1}$ Faculté de sciences juridiques, économique et sociales, Université Mohammed V, Avenue \\ des Nations-Unies, B.P. 721Agdal-Rabat, Maroc
}

\begin{abstract}
Résumé : Notre objectif dans ce travail était d'identifier la relation entre les consommateurs socialement responsables et leur satisfaction en considérant le marketing mix et des éléments tels que l'éthique, l'utilité, l'attractivité et le bouche à oreille comme des éléments moteurs de la décision de ces derniers. À cette fin, nous avons mené une étude empirique qui a commencé par une analyse de la littérature et s'est terminée par une enquête menée auprès de 64 citoyens marocains et personnes vivant au Maroc. Nous avons constaté que les éléments de marketing ont une influence positive sur la satisfaction des consommateurs socialement responsables, $68,75 \%$ des répondants étant satisfaits de leur consommation socialement responsable.
\end{abstract}

Mots-clés : Satisfaction du consommateur, Consommation socialement responsable, Attentes, Comportement, Perception.

Abstract : Our objective in this work was to identify the relationship between the socially responsible consumer and his or her satisfaction considering the marketing mix and elements such as ethics, utility, attraction and word of mouth as driving elements of the latter's decision. For this purpose, we conducted an empirical study starting from a literary analysis and ending with a survey that was carried out among 64 Moroccan citizens and people living in Morocco. We found that marketing elements have a positive influence on the satisfaction of socially responsible consumers, with $68.75 \%$ of respondents being satisfied with their socially responsible consumption.

Keywords : Consumer satisfaction, Socially responsible consumption, Expectations, Behaviour, Perception.

Digital Object Identifier (DOI): https://doi.org/10.52502/ijesm.v1i1.173 


\section{Introduction}

Les entreprises aujourd'hui, font face à un défi incontournable concernant la satisfaction des consommateurs, seulement les consommateurs de nos jours sont responsables, engagés et sensibles aux effets environnementaux, et les conditions des travailleurs qui produisent les produits ou offrent les services. Pour répondre aux questions que cette réalité inévitable nous pose, il y des gestionnaires et des marketeurs qui sont préoccupé par ces éléments et d'autres s'améliorent dans cette affaire car reconnaissant l'importance de l'image social de l'entreprise en ce qui concerne leur positionnement et encore la satisfaction de leurs consommateurs qui est l'élément déterminant de la fidélité des consommateurs. De nos jours, toute entreprise qui se valorise ne doit pas se permettre d'être laissée en arrière, bien au contraire, elle doit viser l'amélioration de ses services, une bonne image d'entreprise, répondant ainsi au besoin de clients fidèles avec la garantie d'acquérir des parts de marché considérables. Aussi, elles doivent s'harmoniser avec le contexte car le marketing change en fonction des contextes, sachant que les clients satisfaits sont des ambassadeurs essentiels dans l'acquisition de nouveaux clients, notez qu'en plus d'être fidèles, ils contribuent au développement de la marque (Kotler, 2017).

Un des problèmes jusqu'ici difficile à maîtriser est l'oscillation des résultats lorsque l'intervention marketing reste la même et malgré cela les résultats se comportent de manière fluctuante. Ce fait nous laisse avec la preuve qu'il existe des variables que le marketing ne peut pas contrôler mais peut influencer.

Le sujet est si important que le monde entier n'échappe pas aux standards du marketing et le sujet incessant est observé en permanence dans diverses plates-formes de recherche, qui sont de nature politique, économique, social, écologique et technologique. Kotler présente la satisfaction comme un produit à vendre et l'expérience que le client aura en tant que service. Ces éléments mentionnés ici, nous montrent que l'amélioration de la satisfaction entraîne un engagement du client qui coïncide avec notre recherche. La seule particularité pour nous, est le type de client car nous savons déjà via Webster que tous les clients ne sont pas responsables (Webster 1975; Kotler et al, p.131 2017).

Nous savons que les études de marketing sont totalement associées aux besoins et nous ne pouvons pas dissocier les besoins du client de la satisfaction du même. Car si le marketing cherche à combler ses besoins, c'est bien la recherche de la satisfaction. Par conséquent la plus grande problématique du marketing est la satisfaction des consommateurs puisque la satisfaction est instable, temporelle et très délicate (Évrard, 1993 : 62 ; Ladhari, 2005).

\subsection{Une contextualisation de l'article}

Pour encadrer notre recherche, nous présenterons d'abord le contexte en synthèse abordant les aspects de la mondialisation, de la technologie et en mettant en évidence les représentations actuelles de la responsabilité sociale.

Compte tenu de l'ampleur de la mondialisation et de son impact en termes de satisfaction des consommateurs, il est exceptionnel de voir à quel point les cultures sont si largement influencées qu'elles entraînent une transformation de la perception des consommateurs.

L'auteur (Stien, 2012) dans son travail nous montre comment la culture a un fort impact sur la satisfaction des consommateurs et l'appréciation d'un produit ou d'un service. Il rapporte que dès la 
prise de décision à la consommation, les valeurs éthiques sont les déterminants d'une satisfaction accrue (Stien, 2012).

En contrepartie, l'auteur Kotler, dans son livre sur le marketing 4.0, nous expose au monde dans lequel les responsables marketing seront confrontés à des clients super informés, plus intelligents et dotés d'une technologie de pointe qui les transforme en personnes plus exigeantes. Et donc la satisfaction est un avantage de l'entreprise dont ils sont satisfaits. Cela deviendrait un levier de fidélité et de renforcement de la fiabilité d'une part pour les marques recommandables et d'autre part, pour les marques non recommandables présentant une vulnérabilité. Ces clients sont caractérisés comme «des jeunes, issus des zones urbaines, appartenant à la classe moyenne, à forte mobilité et connectivité». Le contexte politique se caractérise comme un régulateur, cependant, lorsqu'ils touchent les aspects écologiques de manière irresponsable et sans compromis, cela produit des chocs incontrôlables au niveau sociétal ${ }^{1}$ (Kotler et al., 2017).

Actuellement, nous remarquons que les économistes, gestionnaires, politiciens, sociologues n'arrivent plus à détacher les effets de la satisfaction des consommateurs vis-à-vis de leur entreprise dans leurs réflexions, réalisation et rétroaction. Hoeffler et Keller font appel à la conscience des marketeurs concernant la construction d'une marque forte et le fait de garantir que les clients éprouvent une satisfaction cognitive, affective et conative accompagnée des attitudes appropriées (Hoeffler et Keller, 2002) pour éviter les attitudes évoquées par Sheth et Sisodia qui sont la traduction pure de l'insatisfaction (Sheth et Sisodia, 2005).

Quand nous faisons une rétrospective, nous nous rendons compte à quel point les clients ont changé et nous réalisons qu'avec le temps, et les crises et évolutions technologiques expérimentés par les consommateurs, la satisfaction d'hier ne sera plus la même avec celle d'aujourd'hui ni la même pour demain. D'où la nécessité d'une meilleure étude sur la satisfaction du consommateur socialement responsable ${ }^{2}$. Car, ils sont considérés comme les types de clients qui seront servis par les entreprises à l'avenir « Kotler évoque deux paradoxes qui donnent une représentation pointue à propos de ce sujet :

Marketing 4.0 moving from traditional to digital » (Webster, 1975, Kotler, 2017).

La réalité du monde d'aujourd'hui est caractérisée par des événements inusités. Aussi, nous avons également remarqué que l'accélération de ces événements nous projette vers un avenir imprédictible en ce qui concerne les comportements des clients. Vu qu'ils sortent de plus en plus de ce qui est normal vers une situation alarmante. Par conséquent, seule la consommation socialement responsable considérée de manière engagée peut nous accorder une perspective d'un meilleur avenir et pour cela, nous sommes optimistes. En fait, les principaux contributeurs à ce phénomène sont les changements technologiques et les accélérations «les innovations et NTIC» dont nous sommes témoins. Les travaux de (Dupuich, 2011) soulignent que le comportement des hommes est influencé par les

\footnotetext{
${ }^{1}$ The global risk report 2019, $14^{\text {th }}$ Edition

${ }^{2}$ CSR «Un consommateur responsable est celui qui prend en compte les conséquences publiques de sa consommation privée et qui essaie d'utiliser son pouvoir d'achat pour induire des changements dans la société » Webster, 1975.
} 
innovations et par conséquent le niveau de satisfaction. Car elle a une période de validité très courte par rapport aux années précédentes (Dupuich, 2011). Les psychologues et sociologues se sont retrouvés dans une véritable impasse car le monde avance à une vitesse très rapide et le comportement des hommes ainsi que le niveau de satisfaction sont graduellement courts et pour d'autres très accélérés voir même très éphémères alors que c'est dans la recherche d'une satisfaction durable que les innovations NTIC existent dans le but de faciliter le bien être (Sherry Turkle, 2015³).

Il faut noter que la demande des clients est orientée vers un niveau de satisfaction presque illusoire et les entrepreneurs cherchent à les satisfaire malgré tout. Elle a atteint une dimension qui requiert un encadrement et une révision de valeurs et principes éthiques qui doivent être respectés afin que les clients responsables puissent jouir de leur responsabilité ainsi que des privilèges satisfaisants associés ayant un effet durable. Nous avons mis l'accent sur les lignes précédentes pour démontrer à quel point il est important de revoir la satisfaction des clients dans un monde hyper-dynamique.

La satisfaction des clients n'est pas l'une des tâches les plus aisées à exécuter puisqu'ils ne sont pas différents de toute autre personne ou groupe de personnes et sont accueilli de façons différentes et distinctes, les produits et services fournis sont divers. Dans la psychologie et l'analyse de l'échelle des besoins nous remarquons que les clients sont très difficiles à satisfaire. Cela peut être à cause de l'envie de plus qui existe naturellement dans chaque individu. Pour que la satisfaction soit durable, il faut établir des objectifs progressifs ou permanents afin que la subjectivité des plaisirs recherchés ne génère pas de l'insatisfaction, ce qui représente un postulat scientifique qui soutient ce que nous avançons est la Pyramide du besoin de Maslow. (The Principles of Marketing). L'insatisfaction est fréquente et frustrante surtout parce que les clients anticipent en fonction de leurs attentes le sentiment de réalisation ou de besoin remplis. D'une part, il faut noter qu'en réalité, il y a plus des produits et services passables qui remplissent en totalité le besoin des clients. D'autre part, la satisfaction n'a pas une durée infinie quand il s'agit de produits et services car dans le temps, la valeur perçue se dégrade souvent à cause de la rareté et le plus admirable encore est à cause du fait que le client est arrivé au point de satiété.

Dans nos jours, les entreprises n'ont qu'un seul choix, nous pouvons même dire une obligation qui est celle de présenter une valeur supérieure dans les produits et services qu'ils disposent pour leurs clients cibles par rapport aux concurrents.

\section{Problématique}

Nous pouvons souligner qu'une entreprise avec des clients satisfaits sera bien positionnée sur le marché ou dans une dimension de compétitivité souhaitable. En outre, adhérer au concept selon lequel les consommateurs socialement responsables et satisfaits seront le créneau le plus recherché par les

${ }^{3}$ Turkle, S. (2015). Seuls ensemble. De plus en plus de technologies de moins en moins de relations humaines. Échappée (L'). 
entreprises. Il est considéré comme important de discerner quels sont les facteurs qui collaborent pour que les entreprises obtiennent de telles performances. Avec des consommateurs socialement responsables satisfaits, les entreprises qui se disent socialement responsables sauront quelle rétroaction ils perçoivent dans leurs démarches et les encouragera dans ces démarches dites sociétales. Car lorsque le ROMI (Return on marketing investissement) est positif les entreprises ne cesseront de se réinventer dans les mêmes pratiques principalement à cause de sa contribution dans le progrès de l'entreprise (Solcansky, et Simberova, 2010). Ceci pourrait être une solution dans la modélisation de critères qui contribuent au marketing sociétal des sociétés libérant les voies marketing pour le marketing sociétal, marketing vert ou écologique, marketing responsable, marketing solidaire, marketing éthique, marketing équitable... les connotations sont nombreuses et les contributions seraient de mise (Thiery, 2005)

Les apports théoriques que nous avons rencontrés sont peu généralistes et quelques fois catégoriques. Cependant, les contextes marketing changent, la compréhension aussi et le mode de faire, le marketing tout autant. Par conséquent, résoudre cette problématique apporterait une contribution scientifique et un soutien aux connaissances et théories déjà en place. Donc, la question principale à étudier ici est :

Comment pouvons-nous mesurer l'impact des éléments marketing, des facteurs éthiques et moraux auprès de consommateurs socialement responsable?

- Question-auxiliaire :

"Quels sont les éléments marketing de l'entreprise, facteurs d'éthiques et moraux auprès des consommateurs qui contribuent à leur satisfaction et comment pouvons-nous mesurer leur impact ? » (Riadh Ladhari, 2005)

\subsection{Les objectifs et l'intérêt de l'article}

Notre objectif est d'identifier le rapport existant entre le consommateur socialement responsable et sa satisfaction considérant le mix marketing et les éléments tels que l'Éthique; l'utilité, l'attraction et le bouche à oreille comme des éléments conducteurs de la décision de ces derniers. À partir de là, établir une échelle d'évaluation «efforts que l'entreprise peut consentir pour mieux satisfaire ses consommateur ». Pour ce faire, nous ferons usage d'une analyse quantitative et qualitative. Par conséquent, nous recherchons le rapport entre les variables afin de connaître les classes et ses modalités d'achat et qui peuvent devenir des potentiels cibles, segment ou de niches à exploiter.

L'étude a sa délimitation basée sur le cadre théorique autour de la théorie de la satisfaction du consommateur et du consommateur socialement responsable, qui sont évidents dans un environnement de forte intensité concurrentielle. De même, le domaine d'étude est le marché marocain qui tend à adhérer progressivement à des concepts reflétant les bonnes pratiques de la responsabilité sociale (duale : citoyen et entrepreneurial). «La consommation peut-elle améliorer la satisfaction dans la vie ?» 


\subsubsection{L'intérêt de l'article}

Notre intérêt ici est de contribuer aux recherches précédentes sachant que la satisfaction des consommateurs connaît aussi une évolution ainsi comme leurs comportements, cela est dû aux mutations de consommations. Aussi, l'intérêt qui aura une utilité théorique et pratique d'une part sera la contribution scientifique et de l'autre la modélisation des échelles utiles pour les gestionnaires de marketing.

En ce qui concerne la pertinence sociale et la pratique, cet article cherchera une meilleure expérience pour l'entreprise et les consommateurs en termes de satisfaction pour les deux. D'une part le client aura la satisfaction plus adaptée à son besoin et d'autre part l'entreprise se positionnera dans la pensée du client de manière privilégiée.

En cette occasion, nous allons également, chercher à combler le vide existant en termes de connaissances scientifiques ayant comme appui l'appréciation des consommateurs.

\subsection{Hypothèses}

Dans le but de mieux formuler nos hypothèses nous avons évalués les hypothèses des travaux que nous considérons comme base dans le développement de cet article et nous citons :

Les hypothèses soulevées par Ladhari en 2005 :

- Westbrook 1987 mena une étude empirique dans laquelle il a soulevé l'intégration de réactions affectives pour mieux expliquer la variance de la satisfaction après la consommation du produit ou service.

- Les attentes sont aussi vues comme un levier de la satisfaction car des études montrent que plus grandes sont les attentes du client envers un produit ou service plus élevée est la satisfaction. Les auteurs Anderson, 1973 ; Bearden et Teel, 1983 ; Olivier, 1980 ; Olivier et Linda, 1981 ; Olshavsky et Miller, 1972 ; Tse et Wilton, 1988 ; Westbrook et Reilly, 1983 soulèvent une relation linéaire positive entre les deux.

Les hypothèses les plus récentes soulevées par Martinez et Del Bosque(2013) Chung et al., (2015) telles que :

- La satisfaction du client aura une influence positive sur la fidélité du client ; La perception de la RSE par les clients influence positivement leur satisfaction ;

- L'effet de la RSE sur la satisfaction du client est plus important pour le groupe ayant une perception élevée de l'image de l'entreprise que pour le groupe ayant une perception faible ;

- L'effet de la satisfaction du client sur la fidélité du client est plus important pour le groupe ayant une perception élevée de l'image de l'entreprise que pour le groupe ayant une perception faible.

Quant à nous, nous avons analysé les hypothèses de nos prédécesseurs afin de formuler nos propres hypothèses, qui sont liées au thème que nous sommes en train d'aborder. En ce qui concerne les 
déterminants de la satisfaction, l'auteur Ladhari part du principe que tout processus génère un résultat, qu'il s'agisse de satisfaction ou insatisfaction. Il présente aussi les processus cognitif et affectif, par contre à l'intérieur de chacun d'eux, nous avons des éléments mis en rapport avec la satisfaction.

\subsubsection{Aperçu des variables indépendantes et dépendantes}

Variables : Éléments Marketing: (Produit, place, prix, Promotion, People, Processus, et preuve physique); Satisfaction du consommateur socialement responsable; Utilité-réponses négatives par rapport à l'utilité seront considérées dans le cadre de l'inutilité ; Éthiques ces éléments seront considérés comme : la qualité perçue, l'image de l'entreprise, l'utilité réelle, l'attente du client et la valeur perçue.

Dans le but de vérifier la corrélation entre les variables explicatives et la variable expliqué, nous trouvons qu'elles ont été considérées comme des variables ayant une relation positive, négative et neutre. Cela est factuel quand la qualité du service(ou produit) et la perception du client sont estimées de manière significative. Elles sont plutôt corrélatives pour quelques auteurs comme cité dans les travaux de Suhaniya, et Thusyanthy, (2016).

\subsubsection{Variables indépendantes}

Les éléments marketing auxquels nous faisons référence sont en fait les composants du marketingmix qui ont été à plusieurs fois cités comme aussi des facteurs qu'influence la satisfaction. Le mixmarketing est composé des éléments que nous citons : Produit ; Prix ; Place ; Communication ces derniers sont les plus communs. Toutefois, les éléments tels que le «Processus; People (Personnel de vente) ; Physical évidence seront aussi pris en compte.

Selon Rama Yelkur, 2000, il suggère que le marketing mix n'est pas vraiment le vrai facteur mais plutôt les personnes qui travaillent dans la conception du produit, la commercialisation tarifaire, les conditions données au lieu commercial et la façon de communiquer avec les clients. Donc, il va conclure que la motivation du personnel influence sur le bon déploiement du marketing-mix (Yelkur, 2000).

Magatef (2015) nous précise qu'il y a sûrement la nécessité d'apporter une transformation dans les façons de faire le marketing car les éléments du marketing mix ont une influence indéniable dans la satisfaction du produit ou service jusqu'à la communication (Magatef, 2015). Nous allons chercher à valider ces hypothèses ou à les infirmer :

H1.Les éléments marketing n'ont aucune influence sur la satisfaction des consommateurs socialement responsable.

H2. Les éléments marketing ont une influence positive sur la satisfaction des consommateurs socialement responsables.

Selon les travaux de Helson, 1959 Cardozo, 1965 Olshavsky et Miller, 1972, ils suggèrent que la satisfaction est la conséquence qui résulte des attentes préalables et la performance perçue du produit 
(ou du service). Ce modèle se décompose en trois postulats: a) les attentes préalables du consommateur en rapport avec le produit (ou le service); b) le jugement porté sur la performance du produit (ou du service) au cours de l'expérience de consommation et c) la dis-confirmation (positive, négative ou neutre) qui résulte de la comparaison entre la performance et les attentes (Ladhari,2005). Mais, quand le sujet est socialement responsable, il y a des principes liés aux attentes et des valeurs défendues dans les perceptions. Deux personnes avec des valeurs différentes peuvent percevoir le même produit ou service de façon différente, c'est commun en Hollande, la consommation du porc mais au Maroc c'est un péché. Donc, il y a la question de l'adaptation de produit ou services aux cultures... (Stien, 2012).

1. Le principe associé à la consommation socialement responsable est éloigné du simple principe de l'utilité ${ }^{4}$ il est en soi même le principe de l'éthique et la moralité selon Thiery ${ }^{5}$, 2005. Carrigan et Attalla, 2001 en étudiant les comportements des clients lorsqu'ils jugent leurs façons d'acheter par rapport à l'éthique, ils ont identifié 4 catégories ${ }^{6}$ de clients :

- Les clients compréhensifs et éthiques

- Les clients confus et incertains

- Les clients cyniques et désintéressés

- Les clients inconscients

2. «L'éthique est définie comme la recherche du bien vivre et du bien faire, fondée sur une disposition individuelle à agir de manière constante en vue du bien d'autrui et dans des institutions justes, (Rawls, 1971)».

H3. Les achats éthiques n'ont pas d'influence sur la satisfaction et la confiance des clients.

H4. L'achat éthique a une influence positive sur la satisfaction et la confiance des clients

1. L'utilité vient donner ici une représentation de la valeur morale.

H5. Les achats utiles génèrent une satisfaction client socialement responsable ;

H6. Les achats inutiles influencent la satisfaction du client ;

\footnotetext{
${ }^{4}$ L'utilité qui est la mesure du bien-être ou la satisfaction par la consommation ou encore l'obtention d'un certain nombre de biens et services. Ils l'appellent aussi la valeur morale de la richesse (Nicolas Bernoulli ; Daniel Bernoulli, 1713 ; 1728)

${ }^{5}$ Thiery, P. (2005). Marketing et responsabilité sociétale de l'entreprise: entre civisme et cynisme. Décisions Marketing, 59-69.

${ }^{6}$ Ils ont dégagé quatre catégories de consommateurs : 1. Les compréhensifs et éthiques : font une discrimination entre les entreprises éthiques et non éthiques et sont enclins à répondre positivement au comportement éthique réel des entreprises, 2. Les confus et incertains : voudraient acheter éthique mais ils demeurent déconcertés par le manque d'orientation et les messages contradictoires concernant le comportement éthique des entreprises, 3 . Les cyniques et désintéressés : ne souffrent pas d'un manque d'information mais d'un manque de conviction que les entreprises sont réellement éthiques, 4 . Les inconscients : le manque de connaissance sur l'éthique signifie qu'elle n'est pas encore entrée dans leur équation d'achat. Un accroissement de leur conscience éthique peut engendrer une intention d'achat éthique plus forte. » Amara, A. B. B., \& Zghal, M. (2008). L'impact de la relation «éthique-confiance» sur l'intention d'achat du consommateur. La Revue des Sciences de Gestion, (6), 53-64.
} 
H7. Les achats inutiles n'influencent pas la satisfaction du client.

3. Attraction: elle constitue l'un des enjeux majeurs du marketing car c'est par elle que des actions effectives peuvent être entamées. Kotler, la considère comme l'une de sources essentielles dans le chemin des clients vers l'action ou l'apologie des marques. À l'ère de la connectivité, celle-ci et largement définie par la communauté et non par l'individu (Kotler, 2017). Cependant, nous concevons que l'attraction en marketing est une force gravitationnelle qui rapproche les clients d'un produit ou service par la curiosité liée aux désirs ou besoins latents ou non latents chez l'individu (essai de définition « Carlos Filipe Inglês da Silva, $2021 »)$.

4. Bouche à oreille: est un phénomène de propagation orale d'informations ou avis relatifs à un produit, service ou organisation. Elle peut avoir plusieurs formes d'existence par exemple : les actions de parrainage, le marketing viral, le buzz, le mauvais bouche à oreille (bad buzz) et le bouche à oreille électroniques (B. Bathelot, 2020).

\subsubsection{Les variables dépendantes}

La satisfaction du consommateur socialement responsable sera notre variable dépendante et nous la définissons comme suit: "Un consommateur responsable est celui qui prend en compte les conséquences publiques de sa consommation privée et qui essaie d'utiliser son pouvoir d'achat pour induire des changements dans la société »(Webster, 1975, p.188) ${ }^{7}$.

Nous allons formuler une définition essai de définition : n'ayant pas trouvé une définition proprement dite, nous avons toutefois mis en évidence la définition de Webster qui stipule que la satisfaction du consommateur responsable serait un individu soucieux de contribuer aux changements positifs dans la société. En outre, l'auteur paraphrasant Holbrook, 1994, donne la définition suivante: la consommation socialement responsable est tenue comme une attribution éthique au comportement actif du consommateur dans la poursuite de la moralité orienté vers les autres de manière intrinsèque, dans la mesure où la vertu est sa propre récompense (Holbrook, 1994). Ce qui caractérise la satisfaction des consommateurs socialement responsable peut dégager des différentes définitions de la satisfaction du consommateur dans notre premier travail qui consistait à évaluer. Nous avons fait appel aux raisons qui poussent les étudiants à acheter de manière responsable et les critères tels que : le bien être, la santé, la préservation de l'environnement, la réduction des déchets organiques par le recyclage, et les conditions sociales sont les facteurs auxquels les étudiants s'identifient le plus (Silva et Saad, 2020).

\section{3. État de l'art : « la satisfaction du consommateur socialement responsable »}

Dans le cadre du présent article, nous avons évalué quelques articles pour une meilleure production de connaissance. Ainsi, la satisfaction du consommateur est l'objet de nombreuses études et les questions

\footnotetext{
${ }^{7}$ François-Lecompte Agnès, «La consommation socialement responsable : oui mais... », Reflets et perspectives de la vie économique, 2009/4 (Tome XLVIII), p. 89-98. DOI : 10.3917/rpve.484.0089.
} 
en rapport avec la fidélité et l'image de marque ou de l'entreprise peuvent en être facilement déduites. Notre problématique découle du marketing socialement responsable et le degré de satisfaction du consommateur responsable qui normalement, dans son raisonnement et comportement économique se distingue de ceux n'étant pas préoccupé par l'impact de leur consommation (Webster, 1975; Dampérat et Dussart, 2007 ; Lahdari, 2005).

Swaen et Chumpitaz, (2008) démontrent que la RSE a un impact sur la confiance des consommateurs variable qu'influence directement la perception du consommateur et la satisfaction du consommateur. Également, Lahdari (2005) dans ses études a pu statuer quelques déterminants de la satisfaction faisant appel aux processus cognitifs et affectifs et nous voulons sauvegarder le travail de Aurier et Evrard dans lequel ils s'intéressent à mesurer la satisfaction du consommateur vis-à-vis de l'expérience de consommation (Aurier et Evrard, 1998 ; Ladhari, 2007 ; Swaen et Schumpitaz, 2008).

Les méthodes les plus utilisées dans les études citées ci-dessus, étaient la révision bibliographique et la méthode empirique. Obtenant ainsi des résultats qui expriment un besoin de compréhension approfondie du comportement des consommateurs ainsi que la raison de certains produits, aujourd'hui certains consommateurs sont prêts à le résister étant donné leurs effets néfastes sur la société.

Suite à notre révision de ces travaux notre intérêt s'est accru étant donné que dans le travail de Silva et Saad, sur «la perception des étudiants sur la consommation socialement responsable : une étude empirique au Maroc » nous avons déjà éprouvé une sensibilité de la part des consommateurs socialement responsables (Silva et Saad, 2019). Nous pouvons conclure que des travaux dans ce sens devraient être effectués pour une meilleure compréhension du consommateur responsable et engagé qui, dans les années à venir évoluera davantage.

\subsection{Cadre théorique}

\subsubsection{La satisfaction du consommateur « Différents définitions »}

D'après Cardoso (1965), «les attentes servent directives au consommateur lors du processus de formation de la satisfaction ». Il ajoute aussi qu'elle dépend non seulement du produit mais aussi de l'expérience comprenant l'acquisition du consommateur.

Howard et Seth (1969) énoncent que « c'est l'état d'être récompensé de manière adéquate ou inadéquate dans une situation d'achat pour les sacrifices encourus» Pour Hunt (1977) «c'est l'évaluation rendue que l'expérience était au moins aussi bonne qu'elle était supposée d'être » Evrard (1993), «la satisfaction est une cognition, émotion émanant d'un processus affectif et cognitif, appréhendé dans une perspective transactionnelle ou relationnelle».

«La satisfaction est l'état psychologique, postérieur à l'achat et relatif.» (Evrard, 1993)

Pour Oliver (1996) «la satisfaction comme la réponse à la réalisation des attentes du client, traduisant ainsi une réponse à la qualité produite par le prestataire. Il s'agit de l'évaluation du fait que le service a procuré (ou procure) un niveau d'agrément jugé pertinent ». 
Pour Bartikowski (1999), «La satisfaction est le résultat d'un processus de comparaison psychiques et complexes. La comparaison d'une valeur théorique avec une valeur effective: paradigme de confirmation / infirmation ».

Selon Kotler, Keller et Manceau (2015) «La satisfaction correspond au jugement d'un individu qui compare la performance perçue d'un bien ou d'un service lors de sa consommation avec ses attentes préalables. Si la performance se situe en deçà des attentes, le client est déçu et insatisfait. Si les deux correspondent, il est satisfait. Si la performance va au-delà, il est très satisfait ou même enchanté ».

\subsubsection{L'indispensabilité de la satisfaction du client pour l'entreprise}

La satisfaction du client contribue pour une entreprise au niveau concurrentiel rendant évident la rétroaction des investissements et efforts mis en place par l'entreprise. C'est en fait, une manifestation du résultat final de l'entreprise chez le client que peut se traduire par la fidélité, la rentabilité, l'élargissement des parts de marché, réduction des coûts inutiles, l'auto-évaluation pour s'améliorer et plusieurs autres raisons. Le marketing a comme objectif principal satisfaire les désirs et besoin des clients par les meilleurs produits et services. Donc ce que la satisfaction du client ${ }^{8}$ a comme débouché est irremplaçable. «Sans la satisfaction il n'y aura pas de rentabilité, au contraire les pertes peuvent devenir irréparables » (Keith, 1960 ; Muller, 1991 ; Hennig, Thurau et Hansen, 2013).

Là où il y a des clients insatisfaits, il aura le boycott des produits ou services et une mauvaise image de l'entreprise, une dévalorisation de la marque qui aura comme conséquence la perte de notoriété et des parts de marché, (Hoeffler et Keller, 2002). Selon Muller, 1991 la satisfaction du consommateur favorise la fidélité et permet de gagner et regagner la confiance du client tant que les biens et services sont excellents. D'autre part Ladhari, 2005 pour lui c'est un indicateur qui permet de mieux travailler les outils de la compréhension du comportement du client (Muller,1991; Ladhari, 2005).

\subsubsection{Quel intérêt le marketeur doit accorder à la satisfaction du consommateur ?}

Compte tenu des paragraphes précédents, il est important de noter que cette observation est factuelle, en revanche, Kotler et al, 2017 ne manque pas de l'exposer, peut-être pas de la même manière que

\footnotetext{
${ }^{8}$ The Ten Commandments of good business

1. The customer is the most important person in my business.

2. The customer is not dependent on us; we are dependent on him.

3. A customer is not an interruption of our work; he is the purpose of it.

4. A customer does us a favour when he calls; we are not doing him a favour by serving him.

5 . The customer is part of our business, not an outsider.

6. The customer is not a cold statistic; he is a flesh and blood human being with feelings and emotions like ours.

7. The customer is not someone to argue or match wits with.

8. The customer brings us his wants; it is our job to fill those wants.

9. The customer is deserving of the most courteous and attentive treatment we can give him.

10. The customer is the lifeblood of this, and every other, business. (Kotler, Marketing Principles)
} 
nous l'exposons. Une mise à jour a été faite pour ce qu'il advient de la théorie de Kotler sur l'intensité concurrentielle qu'au départ était énoncée par Adam Smith (Kotler et al, 2017).

Concernant les nouveaux aspects intrinsèquement liés au point de départ de la dimension stratégique, nous pouvons souligner ici que le pouvoir a changé de mains, car les clients ont aujourd'hui une grande influence sur les stratégies, non plus simplement en tant que le «centre » d'étude, mais aussi en tant que «collaborateurs » et les associés stratégiques des entreprises (Kotler, Kartajaya, Setiawan, 2017).

\subsubsection{L'importance que le marketeur doit accorder à la satisfaction pour finir avec l'infortune}

Considérant l'approche, nous dirons alors qu'il s'agit d'une transition dimensionnelle de l'exclusion à l'inclusion. Ce point évoqué dans le livre Marketing 4.0 de Kotler nous amène à comprendre qu'avec le client du côté managérial, les portes sont ouvertes pour que nous sachions mieux quel type de client il est?, Comment le satisfaire s'il est socialement responsable et comment l'encadrer s'il n'est pas le cas.

Du point de vue marché, la dimension est surtout commerciale et tout cela est question de branding et d'élargissement de la part de marché. Nous ne pouvons pas nier que ces éléments apportent une grande tournure financière à l'entreprise, ce qui rend les «stakeholders» plus engagées. En revanche, les mêmes se manifestent prudent quand il s'agit de faire des investissements au niveau de l'axe RSE compte tenu des bénéfices que sont envisagés dans le long terme, comme nous le savons ils préfèrent que les dividendes soient rapides.

\subsubsection{Satisfaction du consommateur : les conséquences de l'ignorance}

Ignorer l'importance de la satisfaction des consommateurs revient au même qu'ignorer les profits que reviendraient à l'entreprise dans le cadre du marketing que ce soit en revenu, au niveau de l'image de l'entreprise, les parts de marché. D'où le besoin d'en parler car elles peuvent générer plusieurs problèmes auxquels les managers ne devraient pas cesser de prêter attention. Pour Ladhari, 2005, il y a trois comportements qui peuvent être disséminés et ces derniers sont; la réclamation, la fidélité et le bouche-à-oreille. Ces derniers ont été distingués en trois catégories : psychologiques, culturelles et démographiques. Pour Bearden, Teel et Crockett, 1980 ; Bernhardt, 1981 ; Gronhaug et Zaltman, 1981 ; Singh, 1990 ; Singh, 1990 ; Farhangmehr et Silva, 1995 ; Hogarth, English et Sharma, 2001, les personnes qui réclament sont des jeunes, des gens bien éduquées et de personnes détenant de revenus considérables donc si nous comprenons bien, ce sont là des clients potentiels et ciblés dans le marché pour cette raison même ils ne doivent pas être ignorés. L'ignorance de plusieurs est un avantage en quelque sorte. Toutefois, à un moment, ils peuvent représenter une menace indéniable pour l'entreprise en s'exprimant par la résistance. D'où l'importance d'éduquer les clients à être et se conduire de manière socialement responsable pour se créer un avenir avantageux auprès d'eux. D'autres sont perçus psychologiquement comme des personnes dont l'attitude est freinée par la 
perception qu'ils seront considérés comme des revendicateurs (Goodwin et Spiggle, 1989 ; Blodgett et Granbois, 1992 ; Richins, 1983). Enfin, une situation culturelle est exposée de telle sorte que les coutumes et les traditions se révèlent différentes entre les peuples, par exemple, les Asiatiques et les Américains sont perçus comme moins grassouillets (Day, Grabicke, Schatzle et Staubach, 1981 ; Richins et Verhage, 1985 ; Foxman, Raven et Stem, 1990).

En premier lieu, Jacoby et Jaccard, 1981 énoncent la réclamation, le départ du client vers une autre entreprise comme des éléments que les entreprises ne doivent sous aucun prétexte ignorer (Bearden et Teel, 1983; Hirschman, 1970; Richins, 1987; Singh, 1990). Ces derniers ont été cités dans le travail de Ladhari, 2005. De leur côté, Richins et folks illustrent que les clients se montrent insatisfaits pour deux facteurs comme la gravité des problèmes, le degré de responsabilité, la performance médiocre du produit ou service et qui est susceptible de se répéter dans l'avenir (Richins, 1987 ; Folks, 1984).

En deuxième lieu, la fidélité n'est rien d'autre que le résultat de la satisfaction qui fera que le client achète à nouveau. En opposition, l'insatisfaction est accompagnée d'infidélité et ce dernier est créé souvent par l'entreprise, chose difficile d'accepter mais une vérité de remise en cause. Le sens de la fidélité change en fonction de son impact direct ou indirect qui est réalisé au travers de la qualité perçue, ceci dit, l'opposée est vraisemblable. (Bitner, 1990; Taylor et Baker, 1994; Fornell et al., 1996; Host et Knie-Andersen, 2004 ;). En fait, l'auteur Coyne et autres, établissent une relation entre fidélité et satisfaction «satisfaction est faible, modérée quand la satisfaction est à un niveau intermédiaire et très fort quand la satisfaction est à un niveau élevé » au travers de ces derniers, ils affirment que la satisfaction est proportionnelle à la fidélité. Si la satisfaction diminue la fidélité aussi et si elle s'accroît, c'est pareil pour la fidélité (Coyne, 1989 ; Ngobo, 1998; Oliva, Oliver et MacMillan, 1992). La fidélité socialement responsable est le fruit de la satisfaction de raison énoncée précédemment dans le premier point.

En troisième lieu, le bouche-à-oreille est un canal du marketing à double tranchant car il peut servir à l'entreprise ou la desservir cela en fonction de la satisfaction ou l'insatisfaction du consommateur. Les auteurs Swan et Oliver, Reichheld et Sasser, 1990; 1989 Ladhari, 2005 annoncent que les clients rendent leur niveau de satisfaction connu souvent de manière informelle c'est -à-dire par le bouche-àoreille. D'après Richins et Bloch en 1986 c'est le moyen par lequel la gravité du problème (insatisfaction) perçu ou l'échec de satisfaire les besoins des clients (Singh, 1990) est exposée à d'autres qui pourraient aussi acheter. Ignorer les effets du bouche-à-oreille aurait un impact néfaste sur l'image de l'entreprise dans le marché, cela serait tel un petit renard que détruit la part du marché. En 2016 l'auteur Mouna Benhallam, présente sa thèse sur « La résistance socialement responsable à la consommation : proposition de conceptualisation et implications pour les pratiques du marketing» et cite une des raisons fondamentales d'échec statué dans ce travail "Cet échec de réponse aux attentes du consommateur remet notamment en cause la capacité du marketing à anticiper, à prospecter les exigences d'individus et de groupes, faisant pourtant partie du marché, d'où l'intérêt pour le marketing d'explorer des formes et manifestations spécifiques de résistance » 
parce que, lorsque le bouche-à-oreille est négatif, il produit effectivement des plaintes, réclamations, boycott et le prosélytisme (résistance active) alors que d'autre côté elle produit la fidélité, propagation de la bonne image, des adeptes de la marque (Benhallam, 2016).

\subsection{La consommation socialement responsable}

La consommation socialement responsable est l'un des enjeux majeurs de l'ère du consumérisme transformée voire diversifiée, elle est un facteur crucial sur le plan économique, social et écologique. Car un consommateur socialement responsable par son vœu de citoyen responsable peut influencer la production et la façon dont les produits sont distribués et/ou livrés. De même, au niveau social, elle peut contribuer à améliorer les conditions de vie et surtout favoriser le bien-être et la santé au niveau écologique. Ces dimensions nous amènent à comprendre comment il a un rôle à jouer dans la durabilité d'un meilleur environnement, tout en procurant un bien-être qui peut être maintenu dans le temps grâce à un engagement continu pour des causes qui bénéficient aux vies et à la société en général.

\subsubsection{L'évidence de la satisfaction dans la consommation responsable}

Tout consommateur qui veut ou voudrait se présenter comme consommateur socialement responsable a en lui une attente et une perception de ce qu'il espère de percevoir en termes de satisfaction lors de la consommation d'un produit ou service socialement responsable. Cette satisfaction tant attendue est souvent caractérisée par la santé, le bien-être, le sentiment d'être écologiste (préserver l'environnement ou d'être les gardiens de la nature), le bonheur, vivre dans une société meilleure. Cijoint quelques définitions de la consommation socialement responsable :

- " Un consommateur qui prend en compte les conséquences publiques de sa consommation privée et qui essaie d'utiliser son pouvoir d'achat pour induire des changements dans la société »Webster, 1975, p.188.

- «La consommation socialement responsable : il s'agit de contribuer au bien-être social et environnemental des autres » Engel et Blackwell, 1982.

- $\quad$ "Individu concerné non seulement par sa satisfaction personnelle mais aussi par le bien-être sociétal et environnemental » Anderson et Cunningham (1972).

- «Individu préoccupé à la fois par son bien-être personnel et le bien-être social » Belch, $1979 ; 1982$.

- «Le consommateur socialement responsable achète des biens et des services qu'il perçoit comme ayant un impact positif sur son environnement et qui utilise son pouvoir d'achat pour exprimer ses préoccupations sociales » Robert, 1995.

- "Individu qui évite d'acheter aux entreprises qui nuisent à la société et qui recherche activement des produits d'entreprises qui aident la société » Mohr, Webb et Harris, 2001. 
- Ce style de vie correspond à la pratique d'une "simplicité volontaire ", c'est à-dire aux actions conformes à l'idée que "la satisfaction personnelle, l'accomplissement de soi et le bonheur résultent d'un engagement avec les aspects non matériels de la vie »Zavestoski, 2002.

- $\quad$ "La consommation socialement responsable est une notion qui inclut tous les comportements de consommation où l'individu prend en compte l'impact de ses achats sur l'environnement et la société en général ». Francois-Lecompte, A. (2006).

Notons, qu'il est évident que les supporteurs de la consommation socialement responsable ont une raison, une cause qu'ils défendent de manière indéniable et collectivement : c'est le bien être, la préservation de l'environnement et la réalisation de soi.

\section{Méthodes - récolte et variables}

L'objectif de ce travail est d'identifier le rapport existant entre le consommateur socialement responsable et sa satisfaction considérant les variables du mix marketing et les éléments tels que l'Éthique ; l'utilité, l'attraction et le bouche à oreille comme de éléments conducteurs de la décision de ce dernier.

Les répondants proviennent de différentes régions du Maroc spécifiquement: Casablanca Settat, Fès-Meknès, l'Oriental, Marrakech-Safi, Rabat-Salé-Kenitra, Souss-Massa. L'enquête que nous avons effectuée a atteint un échantillon de 64 citoyens résidents au Maroc, dont l'âge varie entre 20 et plus de 60 ans avec une capacité financière mensuelle située dans l'intervalle de : moins de 2500 dhs à plus de 10000 dhs.

L'enquête s'est déroulée en ligne via «Google forms » au travers d'un questionnaire (voir annexe 1), qui a permis de relever les raisons de modes de consommation. Pour mieux interpréter toutes les données de cette recherche nous avons illustré sous forme de tableaux et graphiques les données récoltées. En se servant des outils tels que Spss et Excel. Nous avons sélectionné la tranche d'âges et de salaires considérant que ce sont ces personnes ayant une capacité d'exercer leurs décisions sans contrainte et en plus avec un certain bagage en termes de notions de la citoyenneté et la responsabilité sociétale.

Notre positionnement dans cette recherche est interpretativiste. Les interprétativistes, «se concentrent sur des représentations de la réalité traduisant l'expérience de leur relation au monde » (HladyRispal, 2002). Et il est dit que « le chercheur interpretativiste est amené à comprendre et interpréter la réalité en s'interrogeant sur les motivations des acteurs (Gavard-Perret et al., 2008). C'est un positionnement adopté aussi pour comprendre le processus. Nous avons retenu de l'hypothèse 1 à 5 et les hypothèses 6 et 7 qui serviront pour les études ultérieures. Les deux premières restent les principales tandis les autres trois se trouvent dans l'annexe. 
Tableau $\mathbf{n}^{\circ}$ 1: Les variables sociodémographiques par villes

\begin{tabular}{|c|c|c|c|c|c|c|c|c|}
\hline & Ville & $\begin{array}{c}\text { Casablanca- } \\
\text { Settat }\end{array}$ & Fès-Meknès & l'Oriental & $\begin{array}{c}\text { Marrakech- } \\
\text { Safi }\end{array}$ & $\begin{array}{c}\text { Rabat-Salé- } \\
\text { Kénitra }\end{array}$ & $\begin{array}{l}\text { Souss- } \\
\text { Massa }\end{array}$ & $\begin{array}{c}\text { Total } \\
\text { général }\end{array}$ \\
\hline Genre & Féminin & 13 & 7 & 1 & 7 & 4 & $\mathbf{0}$ & 32 \\
\hline \multirow{3}{*}{ Age } & De 20 à 30 ans & 10 & 6 & 1 & 3 & 4 & 0 & 24 \\
\hline & De 30 à 40 ans & 3 & 1 & 0 & 3 & 0 & 0 & 7 \\
\hline & Plus de 40 ans & 0 & 0 & 0 & 1 & 0 & 0 & 1 \\
\hline Genre & Masculin & 8 & 3 & $\mathbf{0}$ & 14 & 5 & 2 & 32 \\
\hline \multirow{3}{*}{ Age } & De 20 à 30 ans & 7 & 3 & 0 & 11 & 2 & 2 & 25 \\
\hline & De 30 à 40 ans & 1 & 0 & 0 & 3 & 2 & 0 & 6 \\
\hline & Plus de 40 ans & 0 & 0 & 0 & 0 & 1 & 0 & 1 \\
\hline
\end{tabular}

Dans ce tableau, nous présentons la population étudiée. Le nombre de personnes ayant participer à l'enquête s'élève au nombre de $\mathbf{6 4}$ résidents au Maroc ; il faut noter les personnes ont répondu en fonction de leur emplacement régionale. Un échantillon qui n'est certes pas considérable, toutefois, il nous permet d'évaluer la tendance de consommation, la valeur ou les principes qui sont associés aux choix des répondants ainsi que le degré de satisfaction éprouvé. Également, nous pouvons observer chez quelques-uns les motivations derrière leurs choix tandis que d'autre sont indifférents aux motifs de leurs achats. 
Tableau $^{\circ} 2$ : TRANCHE SALARIALE PAR RÉGION DE RÉPONDANTS EN \%

\begin{tabular}{|c|c|c|c|c|c|c|c|}
\hline & \multicolumn{5}{|c|}{ TRANCHE SALARIALE PAR RÉGION DE RÉPONDANTS EN \% } & & \\
\hline Salaire & Casablanca-Settat & $\begin{array}{c}\text { Fès- } \\
\text { Meknès }\end{array}$ & l'Oriental & $\begin{array}{c}\text { Marrakech- } \\
\text { Safi }\end{array}$ & $\begin{array}{l}\text { Rabat-Salé- } \\
\text { Kenitra }\end{array}$ & $\begin{array}{l}\text { Souss- } \\
\text { Massa }\end{array}$ & $\begin{array}{c}\text { Total } \\
\text { général }\end{array}$ \\
\hline Moins de $2500 \mathrm{Dhs}$ & $10,94 \%$ & $7,81 \%$ & $1,56 \%$ & $14,06 \%$ & $3,13 \%$ & $0,00 \%$ & $35,94 \%$ \\
\hline $2500-3000 \mathrm{Dhs}$ & $4,69 \%$ & $1,56 \%$ & $0,00 \%$ & $3,13 \%$ & $0,00 \%$ & $3,13 \%$ & $12,50 \%$ \\
\hline 3001-6000 Dhs & $4,69 \%$ & $6,25 \%$ & $0,00 \%$ & $6,25 \%$ & $4,69 \%$ & $0,00 \%$ & $21,88 \%$ \\
\hline 6001-10 $000 \mathrm{Dhs}$ & $12,50 \%$ & $0,00 \%$ & $0,00 \%$ & $7,81 \%$ & $4,69 \%$ & $0,00 \%$ & $25,00 \%$ \\
\hline Plus de $10000 \mathrm{Dhs}$ & $0,00 \%$ & $0,00 \%$ & $0,00 \%$ & $1,56 \%$ & $1,56 \%$ & $0,00 \%$ & $3,13 \%$ \\
\hline Total général & $32,81 \%$ & $15,63 \%$ & $1,56 \%$ & $32,81 \%$ & $14,06 \%$ & $3,13 \%$ & $100,00 \%$ \\
\hline
\end{tabular}

Ce tableau, nous présente la répartition salariale par ville et nous remarquons que parmi les $\mathbf{5}$ catégories de salaire, il y a une (1) catégorie salariale qui a le plus participé ayant des salariés avec de revenus à moins de 2500 Dhs. De plus, elle est la plus considérable avec une participation de 35,94 \% par rapport aux autres catégories. 

Tableau $n^{\circ} 3$ : Niveau de Satisfaction par genre

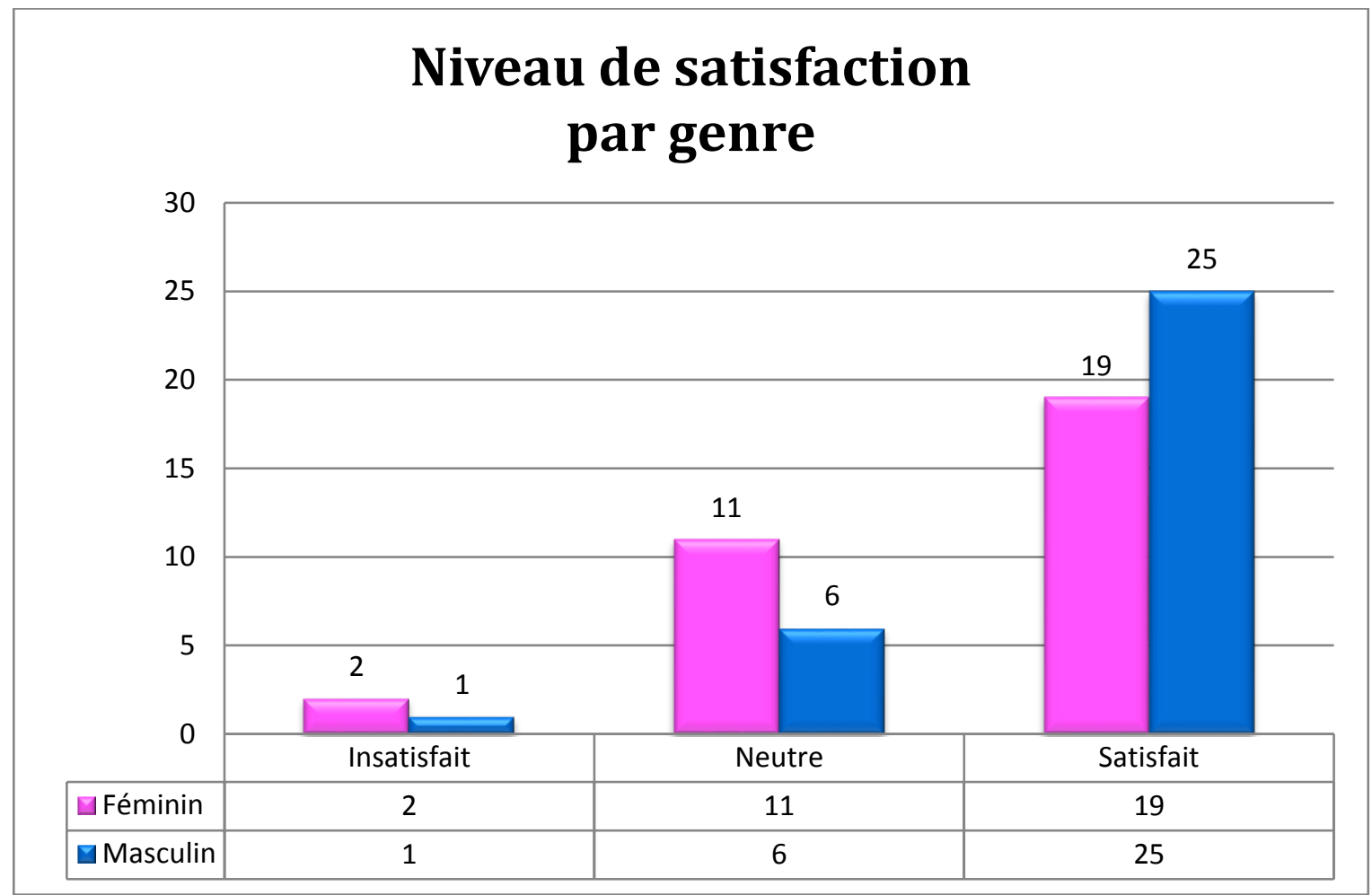

Dans ce tableau nous pouvons remarquer que sur 64 personnes interrogées, 25 sont des hommes satisfaits, contre 19 femmes satisfaites.

Tableau $n^{\circ} 4$ : Mode d'achat

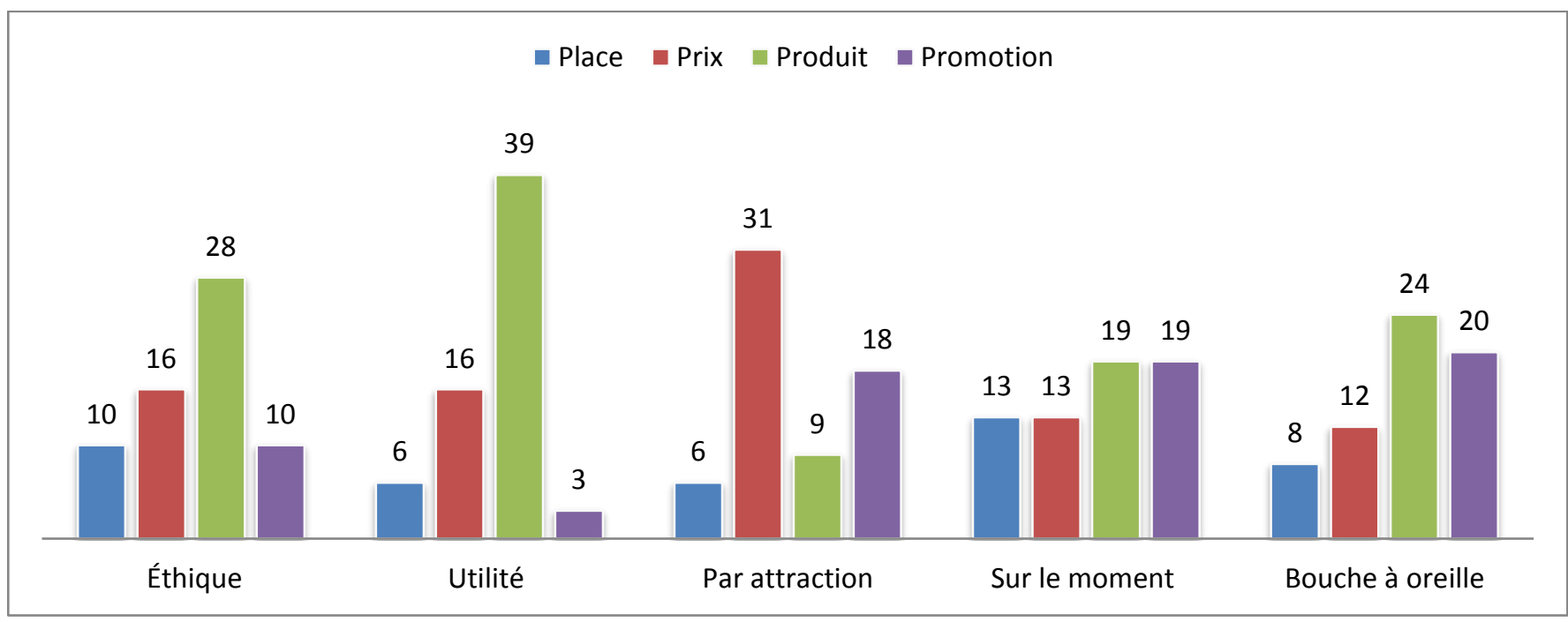

Ce graphique est une représentation des modes de consommation par rapport aux éléments du mix marketing. Il se traduit par une forte adhésion du mode de consommation ayant comme référence le couple produits et utilité. 
Ce couple (produit -utilité) nous révèle une tendance dans les modes d'achats avec 39 personnes parmi 64 qui adhérent. Ce résultat révèle que Thierry n'était pas tout à fait heureux dans son affirmation que l'utilité est dissociable de l'éthique. Car notre résultat renforce plutôt les conclusions des auteurs de Berno ulli (Nicolas et Daniel) qui nous disent que la valeur de la richesse morale se trouve dans l'utilité. Cette étude nous conforte dans la recherche de Carrigan et Attalla, 2001 avec les types de clients précédemment cités. En aucun cas l'éthique n'est remise en cause dans le couple produit-utilité, c'est juste une question théorique qui les sépare, alors qu'en pratique ils sont synergiques.

Tableau n 5 FREQUENCE D'ACHATS SOCIALEMENT RESPONSABLE

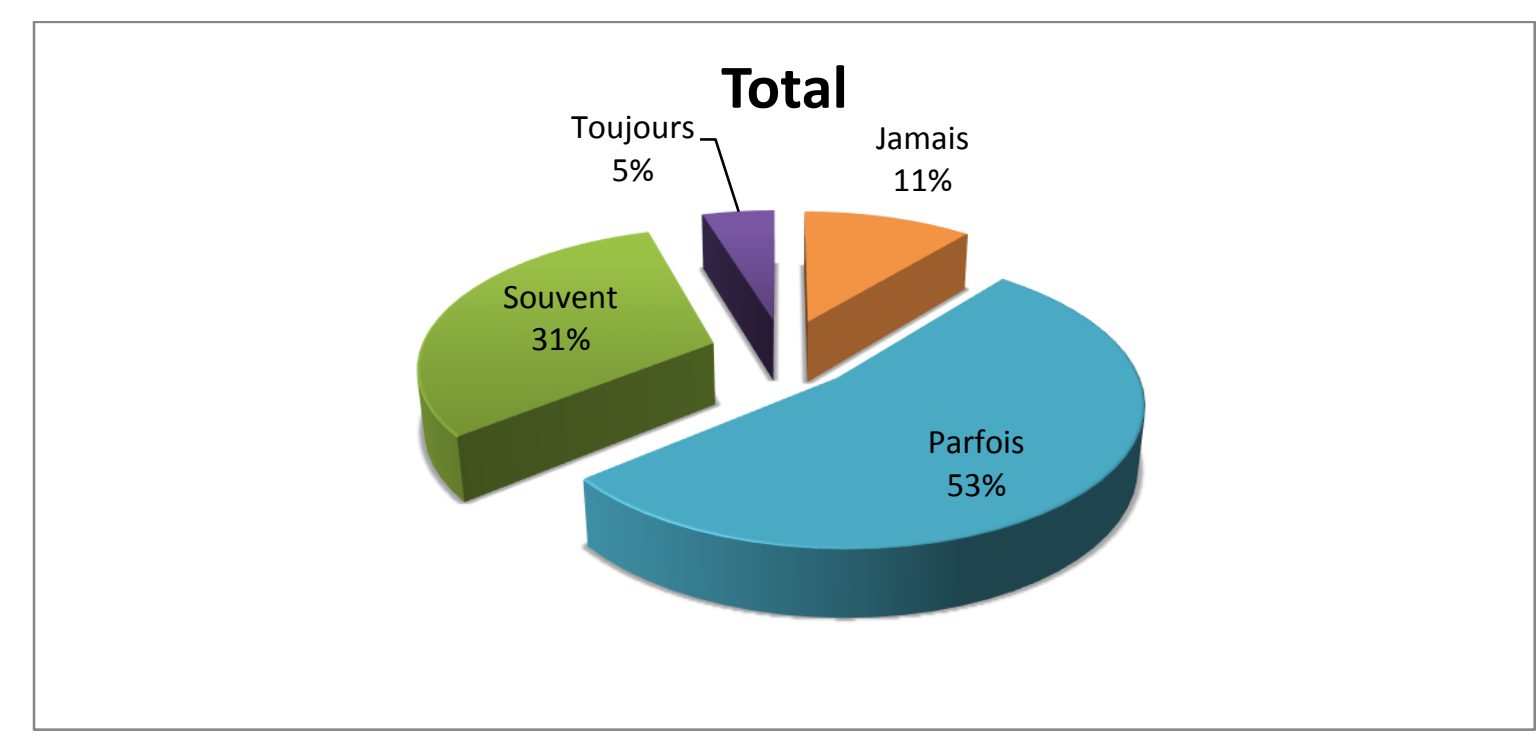

Parmi les personnes interrogées, 53\% des répondants estiment que leurs d'achats sont effectués parfois alors que seulement $5 \%$ de répondants le font toujours. 


\section{Discussion et conclusion}

Considérons que les clients achètent avec l'intérêt d'atteindre un niveau de satisfaction donné, les entreprises à leur tour vendent des produits et des services avec le même intérêt celui de mettre en avant la valeur ajoutée des avantages associés aux produits. Cela, afin d'activer le réachat par la fidélité acquise avec les meilleurs produits.

La satisfaction à long terme est un facteur crucial, surtout lorsqu'il s'agit du consommateur socialement responsable. Lazer 1969 et Thierry 2005 soutiennent que les spécialistes du marketing devraient décider des produits et services à vendre dans une perspective à long terme (Lazer, 1969 ; Thierry, 2005).

Notre étude se concentre sur la satisfaction du consommateur socialement responsable et pour cela nous avons essentiellement cherché à identifier la relation entre le consommateur socialement responsable et sa satisfaction en considérant les variables et éléments du marketing mix tels que l'éthique, l'utilité, l'attraction et le bouche à oreille comme des éléments moteurs de la décision de ce dernier.

Les résultats montrent que $68,75 \%$ des répondants sont satisfaits de leur consommation socialement responsable dont $29,69 \%$ sont des femmes et $39,06 \%$ des hommes, et ils énumèrent certaines raisons de leur satisfaction : "C'est le contrôle que j'ai sur mes choix, leurs apports nutritionnels qu'ils m'apportent, et leur bien-être, et très souvent ma satisfaction est basée et orientée vers la simplicité et la longévité des produits et services. » Un autre répondant «Je dirais que c'est une question d'habitude étant donné que je viens récemment de me lancer dans la consommation de ces produits, je dois prendre le temps de m'habituer» le motif pour ce dernier est «Le fait que les produits répondent à mes attentes, explique ma satisfaction. De même, le rapport qualité-prix abordable est la raison de ma satisfaction des produits que je consomme ». Les études sur la satisfaction du consommateur socialement responsable au Maroc sont rares, cependant, des études proches comme celle de Kobi et Oukassi, 2019 nous présentent ces modes de consommation comme une réponse aux conditions de travail ainsi qu'à la qualité de vie (Kobi et Oukassi, 2019), en retour pour Moussaid et BenMoussa, 2019 souvent les consommateurs qui choisissent de réaliser une satisfaction socialement responsable sont composée de deux classes des traditionalistes (âgés de plus de 50 ans) et les attentifs ne dépassent pas 50 ans et les actifs ( Moussaid et BenMoussa, 2019).

Nous pouvons ajouter que, peu de travaux ont été explorés dans ce sens sauf ceux spécifiés dans les produits alimentaires et cosmétiques (Les antécédents de l'attitude du consommateur marocain envers les aliments fonctionnels : une approche qualitative exploratoire) Moussaid et BenMoussa, 2019; (Les valeurs de consommation des produits biologiques et " Beldi " au Maroc) Jazi, 2014. Une étude qui met en évidence le taux d'insatisfaction est celle réalisée par Kharifi, Berrada et Adyoubah, 2018 sur le 
thème (l'impact du greenwashing sur la satisfaction du client vert, la qualité verte perçue et le bouche à oreille vert).

$\mathrm{Au}$ travers de notre recherche l'hypothèse $\mathbf{H 1}$ est réfuté et la $\mathbf{H 2}$ est confirmé. En effet, nous constatons que les éléments marketing jouent un rôle prépondérant dans la satisfaction du consommateur, surtout lorsqu'il est directement lié au produit, au prix, à la localisation et à la promotion. Ils participent à la satisfaction de l'achat à la consommation et au maintien du plaisir ressenti, qu'il soit cognitif, affectif ou conatif. Les réponses de nos répondants parlent d'elles-mêmes :

- cognitif «Le fait que les produits répondent à mes attentes, explique ma satisfaction. De même, le rapport qualité-prix abordable est la raison de ma satisfaction des produits que je consomme ».

- affectif « parce que le produit saint est nécessaire pour mon bien être; La sensation de contribuer à quelque chose d'éthique »

- Conatif "La connaissance à l'avance de ce que je désire par rapport à ce que je sais du produit»

Nous avons vérifié et attesté à travers les hypothèses $\mathbf{H 4}$ et $\mathbf{H 5}$ que la relation entre l'éthique, l'utilité et la satisfaction est très forte. Si nous examinons le tableau des couples (éthique - satisfaction) et (utilité - satisfaction) ${ }^{9}$, nous pouvons constater que les répondants de cette catégorie sont principalement satisfaits du produit, de sorte que les méthodes dites d'achat impulsif et de bouche-àoreille sont relativement stables dans tous les éléments du marketing mix. Collectivement, ils forment l'ensemble des répondants satisfaits par une analogie de croisement des variables dépendantes avec les variables indépendantes renforçant notre perception et notre croyance dans la relation entre satisfaction et éthique. Cette relation de satisfaction est relativisée selon la modalité d'achat et variée selon les éléments du marketing. Par conséquent, nous réfutons à travers l'analyse l'inefficacité de l'influence des variables indépendantes, puisqu'elles apparaissent comme des éléments sensibles dans les choix, la motivation et le taux de satisfaction des répondants.

L'un des travaux qui soutiennent cette même observation est celui de Lichtlé, et al. 2016 et ils sont allés jusqu'à présenter l'état de satisfaction dans différents compartiments soutenus par la littérature concernant les éléments de la qualité de vie qui sont dimensionnés par l'auteur au niveau tel que : la vie sociale, la vie professionnelle, la vie familiale, la vie spirituelle, la santé ou l'état de ses finances (Diener, 1984 ; Sirgy, 2002). Notre travail nous permet de comprendre que pour atteindre un niveau de satisfaction, il est nécessaire de défendre certaines valeurs dans les habitudes d'achat afin que celles-ci puissent générer la satisfaction attendue. Par conséquent, le client devient fidèle à l'entreprise car il est

${ }^{9}$ Voir les annexes 
satisfait et cela ne peut pas non plus être généralisé car il existe des clients satisfaits qui finissent toujours par changer de fournisseur.

Nous pouvons conclure entre autres que la satisfaction du consommateur est une étude qui peut être développée davantage. Aussi, notre résultat nous a convaincu que la satisfaction du consommateur n'est pas une question qui est toujours prise pour acquise et donc le marketeur devra défendre certains principes et valeurs. En vertu de l'exploitation des hypothèses, nous avons compris que la satisfaction du consommateur dépend de l'engagement du fournisseur, vendeur ou prestataire de services envers les causes qui sont liées aux coutumes et à la valeur attachée à l'utilité du produit et du service rendu au client. Les éléments de marketing ont été exploités au-delà de l'objectif qui nous a été donné. Nous comprenons que la satisfaction du consommateur devra être étudiée davantage pour mieux discerner les comportements des clients, c'est là ce que ce document révèle comme impératif à travers les données que nous avons obtenues. Et ceci est le résultat des hypothèses que nous avons testées et des déclarations qui ont pu être faites lors de la rédaction du présent article.

En sommes, nous proposons que ces études soient affinées (rendues plus pointues) dans le même sens afin de faire émerger des segments ciblés et atteignables au moindre coût avec une optimisation des résultats attendus tout en respectant le bien-être et la satisfaction du consommateur.

\section{Discussion et conclusion}

Considérons que les clients achètent avec l'intérêt d'atteindre un niveau de satisfaction donné, les entreprises à leur tour vendent des produits et des services avec le même intérêt celui de mettre en avant la valeur ajoutée des avantages associés aux produits. Cela, afin d'activer le réachat par la fidélité acquise avec les meilleurs produits.

La satisfaction à long terme est un facteur crucial, surtout lorsqu'il s'agit du consommateur socialement responsable. Lazer 1969 et Thierry 2005 soutiennent que les spécialistes du marketing devraient décider des produits et services à vendre dans une perspective à long terme (Lazer, 1969 ; Thierry, 2005).

Notre étude se concentre sur la satisfaction du consommateur socialement responsable et pour cela nous avons essentiellement cherché à identifier la relation entre le consommateur socialement responsable et sa satisfaction en considérant les variables et éléments du marketing mix tels que l'éthique, l'utilité, l'attraction et le bouche à oreille comme des éléments moteurs de la décision de ce dernier.

Les résultats montrent que $68,75 \%$ des répondants sont satisfaits de leur consommation socialement responsable dont $29,69 \%$ sont des femmes et 39,06\% des hommes, et ils énumèrent certaines raisons de leur satisfaction : "C'est le contrôle que j'ai sur mes choix, leurs apports nutritionnels qu'ils m'apportent, et leur bien-être, et très souvent ma satisfaction est basée et orientée vers la simplicité et la longévité des produits et services. » Un autre répondant «Je dirais que c'est une question 
d'habitude étant donné que je viens récemment de me lancer dans la consommation de ces produits, je dois prendre le temps de m'habituer» le motif pour ce dernier est «Le fait que les produits répondent à mes attentes, explique ma satisfaction. De même, le rapport qualité-prix abordable est la raison de ma satisfaction des produits que je consomme ». Les études sur la satisfaction du consommateur socialement responsable au Maroc sont rares, cependant, des études proches comme celle de Kobi et Oukassi, 2019 nous présentent ces modes de consommation comme une réponse aux conditions de travail ainsi qu'à la qualité de vie (Kobi et Oukassi, 2019), en retour pour Moussaid et BenMoussa, 2019 souvent les consommateurs qui choisissent de réaliser une satisfaction socialement responsable sont composée de deux classes des traditionalistes (âgés de plus de 50 ans) et les attentifs ne dépassent pas 50 ans et les actifs ( Moussaid et BenMoussa, 2019).

Nous pouvons ajouter que, peu de travaux ont été explorés dans ce sens sauf ceux spécifiés dans les produits alimentaires et cosmétiques (Les antécédents de l'attitude du consommateur marocain envers les aliments fonctionnels : une approche qualitative exploratoire) Moussaid et BenMoussa, 2019 ; (Les valeurs de consommation des produits biologiques et " Beldi " au Maroc) Jazi, 2014. Une étude qui met en évidence le taux d'insatisfaction est celle réalisée par Kharifi, Berrada et Adyoubah, 2018 sur le thème (l'impact du greenwashing sur la satisfaction du client vert, la qualité verte perçue et le bouche à oreille vert).

$\mathrm{Au}$ travers de notre recherche l'hypothèse $\mathbf{H 1}$ est réfuté et la $\mathbf{H 2}$ est confirmé. En effet, nous constatons que les éléments marketing jouent un rôle prépondérant dans la satisfaction du consommateur, surtout lorsqu'il est directement lié au produit, au prix, à la localisation et à la promotion. Ils participent à la satisfaction de l'achat à la consommation et au maintien du plaisir ressenti, qu'il soit cognitif, affectif ou conatif. Les réponses de nos répondants parlent d'elles-mêmes :

- cognitif «Le fait que les produits répondent à mes attentes, explique ma satisfaction. De même, le rapport qualité-prix abordable est la raison de ma satisfaction des produits que je consomme ».

- affectif « parce que le produit saint est nécessaire pour mon bien être; La sensation de contribuer à quelque chose d'éthique »

- Conatif «La connaissance à l'avance de ce que je désire par rapport à ce que je sais du produit »

Nous avons vérifié et attesté à travers les hypothèses $\mathbf{H 4}$ et $\mathbf{H 5}$ que la relation entre l'éthique, l'utilité et la satisfaction est très forte. Si nous examinons le tableau des couples (éthique - satisfaction) et (utilité - satisfaction) ${ }^{10}$, nous pouvons constater que les répondants de cette catégorie sont principalement satisfaits du produit, de sorte que les méthodes dites d'achat impulsif et de bouche-à-

${ }^{10}$ Voir les annexes 
oreille sont relativement stables dans tous les éléments du marketing mix. Collectivement, ils forment l'ensemble des répondants satisfaits par une analogie de croisement des variables dépendantes avec les variables indépendantes renforçant notre perception et notre croyance dans la relation entre satisfaction et éthique. Cette relation de satisfaction est relativisée selon la modalité d'achat et variée selon les éléments du marketing. Par conséquent, nous réfutons à travers l'analyse l'inefficacité de l'influence des variables indépendantes, puisqu'elles apparaissent comme des éléments sensibles dans les choix, la motivation et le taux de satisfaction des répondants.

L'un des travaux qui soutiennent cette même observation est celui de Lichtlé, et al. 2016 et ils sont allés jusqu'à présenter l'état de satisfaction dans différents compartiments soutenus par la littérature concernant les éléments de la qualité de vie qui sont dimensionnés par l'auteur au niveau tel que : la vie sociale, la vie professionnelle, la vie familiale, la vie spirituelle, la santé ou l'état de ses finances (Diener, 1984 ; Sirgy, 2002). Notre travail nous permet de comprendre que pour atteindre un niveau de satisfaction, il est nécessaire de défendre certaines valeurs dans les habitudes d'achat afin que celles-ci puissent générer la satisfaction attendue. Par conséquent, le client devient fidèle à l'entreprise car il est satisfait et cela ne peut pas non plus être généralisé car il existe des clients satisfaits qui finissent toujours par changer de fournisseur.

Nous pouvons conclure entre autres que la satisfaction du consommateur est une étude qui peut être développée davantage. Aussi, notre résultat nous a convaincu que la satisfaction du consommateur n'est pas une question qui est toujours prise pour acquise et donc le marketeur devra défendre certains principes et valeurs. En vertu de l'exploitation des hypothèses, nous avons compris que la satisfaction du consommateur dépend de l'engagement du fournisseur, vendeur ou prestataire de services envers les causes qui sont liées aux coutumes et à la valeur attachée à l'utilité du produit et du service rendu au client. Les éléments de marketing ont été exploités au-delà de l'objectif qui nous a été donné. Nous comprenons que la satisfaction du consommateur devra être étudiée davantage pour mieux discerner les comportements des clients, c'est là ce que ce document révèle comme impératif à travers les données que nous avons obtenues. Et ceci est le résultat des hypothèses que nous avons testées et des déclarations qui ont pu être faites lors de la rédaction du présent article.

En sommes, nous proposons que ces études soient affinées (rendues plus pointues) dans le même sens afin de faire émerger des segments ciblés et atteignables au moindre coût avec une optimisation des résultats attendus tout en respectant le bien-être et la satisfaction du consommateur. 


\section{BIBLIOGRAPHIE}

[1] Aurier, P., \& Evrard, Y. (1998). Élaboration et validation d'une échelle de mesure de la satisfaction des consommateurs. In Actes du colloque AFM Bordeaux (pp. 51-72).

[2] Bartikowski, B. (1999). La satisfaction des clients dans les services: une vue situationnelle du poids fluctuant des éléments.

[3] Cardozo, R. N. (1965). An experimental study of customer effort, expectation, and satisfaction. Journal of marketing research, 2(3), 244-249.

[4] Dampérat, M., \& Dussart, C. (2007). Un marketing bien trop vert. Décisions Marketing, 101-106.

[5] Dampérat, M., \& Dussart, C. (2007). Un marketing bien trop vert. Décisions Marketing, 101-106.

[6] Dupui ch, F. (2011). L'émergence des compétences collectives, vers une gestion durable. Gestion 2000, 28(2), 107-125.

[7] Francois-Lecompte, A. (2006). La consommation socialement responsable: proposition d'un modèle intégrateur. Actes de 22ème congrès international de l'Association Française du Marketing.

[8] Hennig-Thurau, T., \& Hansen, U. (Eds.). (2013). Relationship marketing: Gaining competitive advantage through customer satisfaction and customer retention. Springer Science \& Business Media.

[9] Hoeffler, S., \& Keller, K. L. (2002). Building brand equity through corporate societal marketing. Journal of Public Policy \& Marketing, 21(1), 78-89.

[10] Kotler, P., Kartajaya, H., \& Setiawan, I. (2017). Marketing 4.0: Moving from traditional to digital.

[11] Kotler, P., Keller, K. L., Manceau, D., \& Hémonnet-Goujot, A. (2015). Marketing management (Vol. 14). Englewood Cliffs, NJ: Prentice Hall. Kiley, D.(2005). Hey advertisers, TiVo is your friend. BusinessWeek, 17, 97-8.

[12] Ladhari, R. (2005). La satisfaction du consommateur, ses déterminants et ses conséquences. Revue de l'Université de Moncton, 36(2), 171-201.

[13] Lichtlé, M. C., Plichon, V., \& Grzeskowiak, S. (2016). La consommation peut-elle améliorer la satisfaction dans la vie?. Management Avenir, (1), 77-94.

[14] Magatef, S. G. (2015). The impact of tourism marketing mix elements on the satisfaction of inbound tourists to Jordan. International Journal of Business and Social Science, 6(7), 41-58.

[15] Martínez, P., \& Del Bosque, I. R. (2013). CSR and customer loyalty: The roles of trust, customer identification with the company and satisfaction. International Journal of Hospitality Management, 35, 89-99.

[16] Sheth, J. N. (1969). Consumer image and attitude. JMR, Journal of Marketing Research (pre-1986), 6(000003), 381.

[17] Solcansky, M., \& Simberova, I. (2010). Measurement of marketing effectiveness. Economics and management, 15, 755-759.

[18] Stien, E. (2012). L'impact de la culture sur le comportement de consommation: modélisation d'un comportement de consommation éthique ethnique (Doctoral dissertation, Artois). 
[19] Suhaniya, A., \& Thusyanthy, V. (2016). A review on the relationship variables to customer satisfaction. Global Journal of Management and Business Research: E Marketing, 16(5), 87-91.

[20] Swaen, V., \& Chumpitaz, C. R. (2008). L'impact de la responsabilité sociétale de l'entreprise sur la confiance des consommateurs. Recherche et Applications en Marketing (French Edition), 23(4), 7-35.

[21] Thiery, P. (2005). Marketing et responsabilité sociétale de l'entreprise: entre civisme et cynisme. Décisions Marketing, 59-69.

[22] Webster Jr, F. E. (1975). Determining the characteristics of the socially conscious consumer. Journal of consumer research, 2(3), 188-196.

[23] J. Clerk Maxwell, A Treatise on Electricity and Magnetism, 3rd ed., vol. 2. Oxford: Clarendon, 1892, pp.68-73.

[24] I. S. Jacobs and C. P. Bean, "Fine particles, thin films and exchange anisotropy," in Magnetism, vol. III, G. T. Rado and H. Suhl, Eds. New York: Academic, 1963, pp. 271-350. 
7. Questionnaire

A. Genre

○ Masculin; Féminin

B. Votre âge est parmi les

- moins de 20 ans

- De 20 à 30 ans

- De 30 à 40 ans

- Plus de 40 ans

C. Géolocalisation (Par région)
- Casablanca-Settat
- Rabat-Salé-Kénitra Marrakech-Safi
- Fès-Meknès
- Béni Mellal-Khénifra
- Tanger-Tétouan-Al Hoceïma
- l'Oriental
- Drâa-Tafilalet
- Souss-Massa
- Guelmim-Oued Noun
- Laâyoune-Sakia El Hamra
- Dakhla-Oued Ed Dahab

D. Quelle est votre tranche Salariale (bourse ou épargne mensuelle)?
- Moins de 2500 dhs
- $2500-3000$ Dhs
- 3001-6000 Dhs
- 6001-10000 Dhs
- Plus de 10000 Dhs

E. Comment faites-vous vos achats ? En fonction de : (par exemple un produit que tu trouves éthique, utile, attractif, conseillé par quelqu'un....)

\begin{tabular}{|c|c|c|c|c|}
\hline & Produit & Prix & Place & Promotion \\
\hline Éthique & - & - & - & - \\
\hline Utilité & - & - & - & - \\
\hline Par attraction & - & - & - & - \\
\hline Sur le moment & - & - & - & - \\
\hline Bouche à oreille & - & - & - & - \\
\hline
\end{tabular}


F. À quelle fréquence les produits ou services socialement responsables répondent-ils à vos attentes? (Échelle de Linkert)
○ Jamais
- Parfois
- Souvent
○ Toujours

G. Avez-vous une raison particulière ?

H. Êtes-vous satisfait des produits que vous consommez ?
- Neutre
- Insatisfait
- Satisfait
- Satisfait

I. Qu'est-ce qui expliquerait votre satisfaction? 
8. Annexes

Tableau n ${ }^{\circ} 6$ RELATION : ETHIQUE ET SATIFACTION

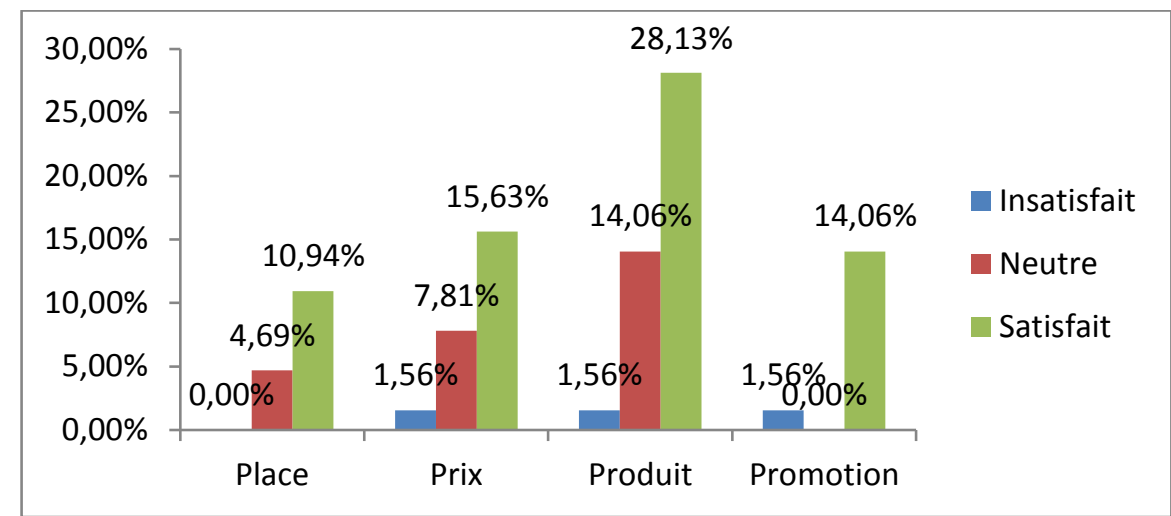

La satisfaction au niveau du mode d'achat du couple (Éthique - Mix marketing) est expliquée à raison de $28,13 \%$ en fonction des produits.

Tableau $n^{\circ} 7$ RELATION : UTILITE ET SATIFACTION

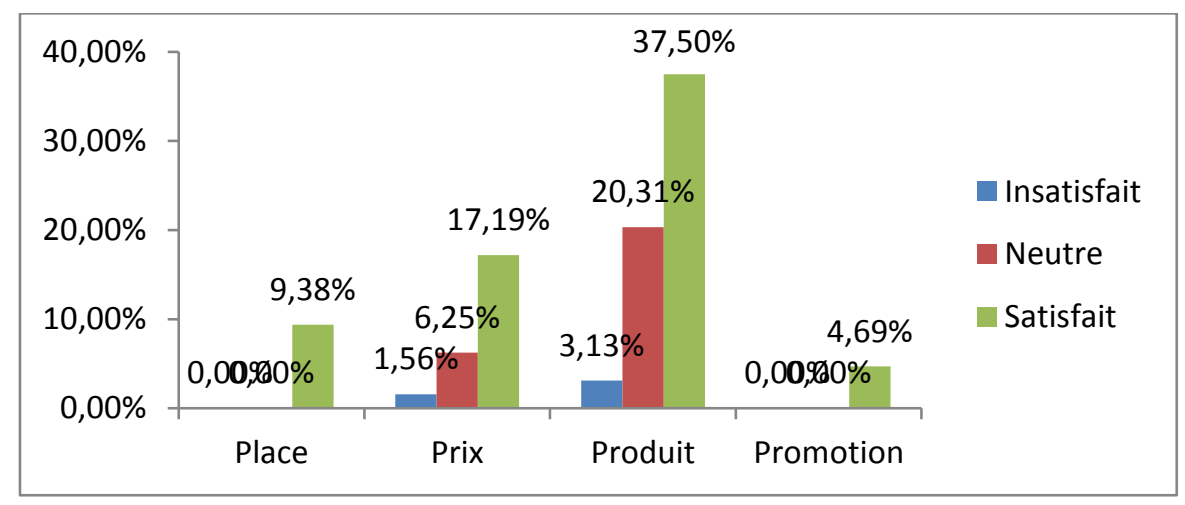

La satisfaction au niveau du mode d'achat du couple (Utilité - Mix marketing) est expliquée à raison de $37,50 \%$ en fonction des produits. 
Tableau n 8 RELATION : ATTRACTION ET SATIFACTION

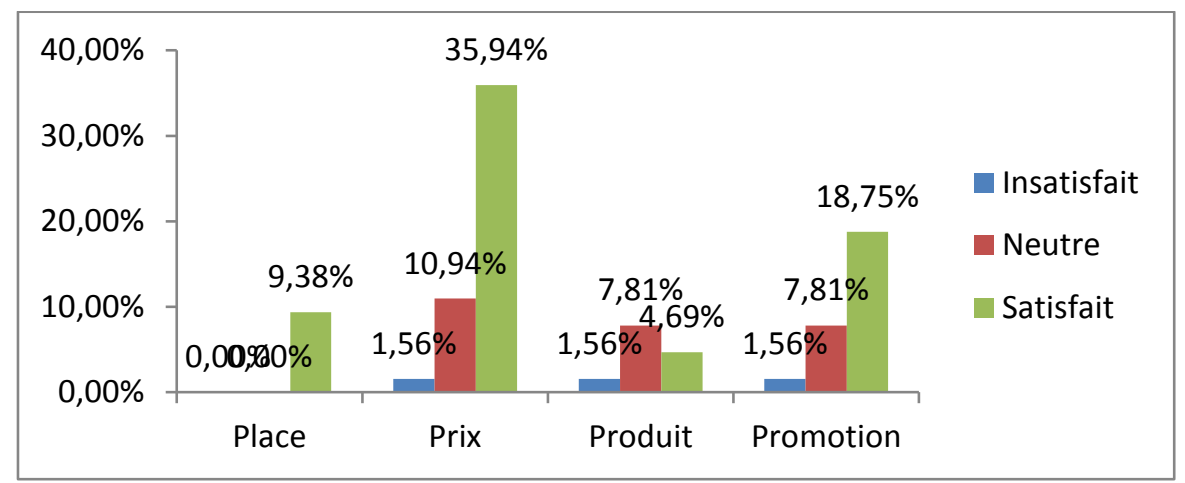

La satisfaction au niveau du mode d'achat du couple (Attraction - Mix marketing) est expliquée à raison de 35,94\% en fonction des prix.

\section{Tableau $n^{\circ} 9$ RELATION : SUR LE MOMENT ET SATIFACTION}

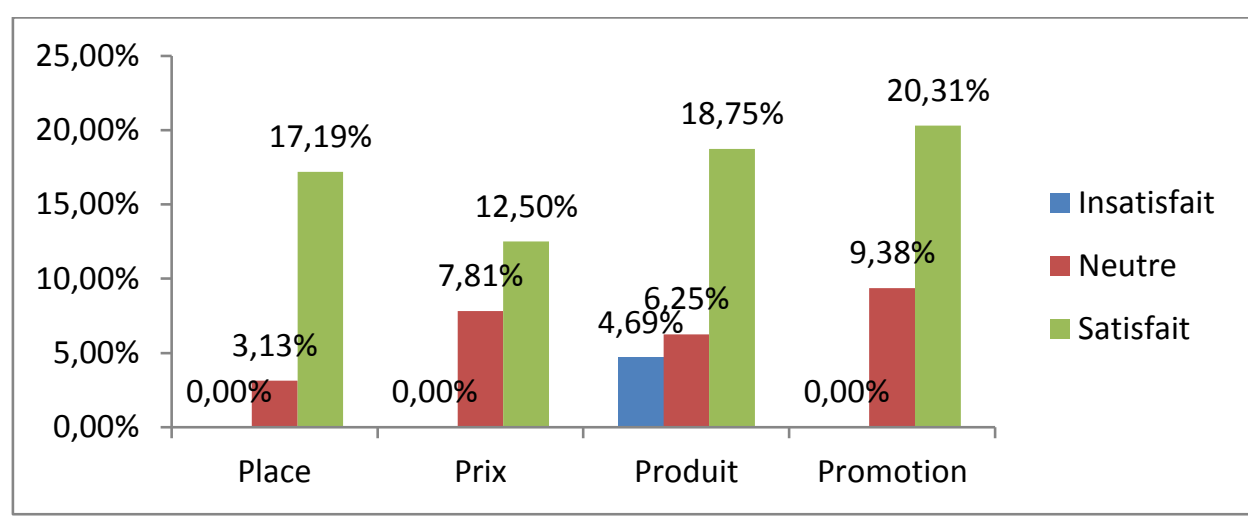

La satisfaction au niveau du mode d'achat du couple (Achat sur le Moment - Mix marketing) est expliquée à raison de $20,31 \%$ en fonction des promotions.

\section{Tableau n ${ }^{\circ} 10$ RELATION : BOUCHE À OREILLE ET SATIFACTION}

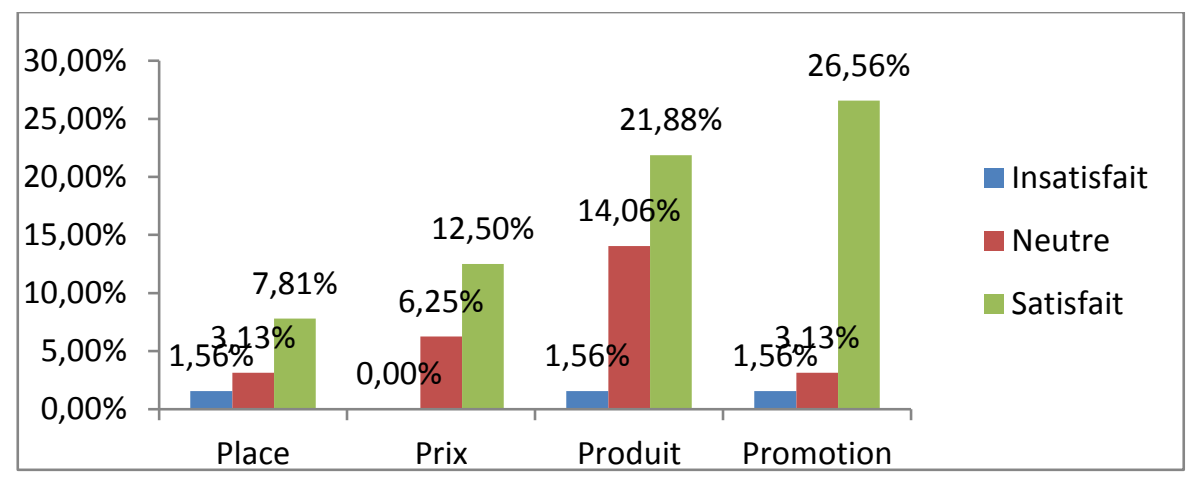

La satisfaction au niveau du mode d'achat du couple (Bouche à oreille - Mix marketing) est expliquée à raison de $26,56 \%$ en fonction des promotions. 